XVIII International Seminar on Health, Food and Human Nutrition

Corresponding Author:

Lino Arturo Rojas Pérez

linoarojas@hotmail.com

Published: 9 September 2021

Production and Hosting by Knowledge E

(c) Lino Arturo Rojas Pérez et al. This article is distributed under the terms of the Creative Commons Attribution License, which permits unrestricted use and redistribution provided that the original author and source are credited.

\section{Effectiveness of the Intrauterine Compression Balloon in Postpartum Hemorrhage, Riobamba Provincial Teaching Hospital - October 2015 to September 2016}

\section{Efectividad del Balón de Compresión Intrauterino en Hemorragia Posparto. Hospital Provincial Docente de Riobamba - Octubre 2015 a Septiembre 2016}

\author{
Lino Arturo Rojas Pérez ${ }^{1}$, Lino Arturo Rojas Cruz ${ }^{1,2}$, María Daniela Villagómez \\ Vega $^{2}$, Augusto Ernesto Rojas $\mathrm{Cruz}^{2,3}$, and Andrés Eduardo Rojas Cruz ${ }^{2}$ \\ ${ }^{1}$ Facultad de Salud Pública, Carrera de Medicina, Facultad de Salud Pública, Escuela Superior \\ Politécnica de Chimborazo, Riobamba, Ecuador \\ ${ }^{2}$ ProSalud Medical Center, Jacinto Gonzales 1951 y Rey Cacha, Riobamba, Ecuador \\ ${ }^{3}$ Centro de Salud de Chocaví, Zona 3, Distrito Guano- Penipe, Ministerio de Salud Pública, \\ Chocaví, Guano, Ecuador
}

\section{Abstract}

Introduction: Postpartum hemorrhage is blood loss greater than $500 \mathrm{~mL}$ after a normal delivery or more than $1000 \mathrm{~mL}$ after a cesarean section, or any postpartum bleeding regardless of the amount causing hemodynamic instability. The uterine blood flow in a term pregnancy is approximately 500 to $700 \mathrm{~mL} / \mathrm{min}$, for this reason, a patient who presents this complication, in a few minutes, can be drained and compromise her life. Objective: To determine the efficacy of the intrauterine compression, balloon in the management of postpartum hemorrhage due to hypotonia or uterine atony, at the General Teaching Provincial Hospital of Riobamba from October 2015 to September 2016. Methodology: The effectiveness of the use of the intrauterine compression balloon after the failure of pharmacological management was analyzed and statistical evaluation was applied to determine the efficacy of this method for the management of this pathology. Results: It was placed in 23 patients who presented postpartum hemorrhage due to hypotonia or uterine atony, and it had an effectiveness of $94.4 \%$ after a vaginal delivery and $80 \%$ after a cesarean section. Reaffirming that it is an excellent option in the management of this type of complications. Conclusions: Postpartum hemorrhage is the second cause of maternal death nationwide, technical and timely action will help reduce maternal morbidity and mortality that this pathology can present; the use of the intrauterine hydrostatic compression balloon is an excellent alternative prior to the surgical management of this pathology.

Keywords: postpartum hemorrhage, uterine bleeding, uterine tamponade with balloon.

\section{Resumen}

Introducción: La hemorragia posparto es la pérdida sanguínea mayor de $500 \mathrm{~mL}$ luego de un parto normal o más de $1000 \mathrm{~mL}$ luego de una cesárea, o cualquier sangrado posparto independientemente de la cantidad que cause inestabilidad hemodinámica. El riego sanguíneo 
uterino en un embarazo a término es de aproximadamente 500 a $700 \mathrm{~mL} / \mathrm{min}$ por tal motivo, una paciente que presente esta complicación, en pocos minutos puede quedar exangüe y comprometer si vida. Objetivo: Determinar la eficacia del balón de compresión intrauterino en el manejo de la hemorragia posparto por hipotonía o atonía uterina, en el Hospital Provincial General Docente de Riobamba de Octubre del 2015 a septiembre del 2016. Metodología: Se analizó la efectividad del uso del balón de compresión intrauterina luego del fracaso del manejo farmacológico y se aplicó la evaluación estadística para determinar la eficacia de este método para el manejo de esta patología. Resultados: Se colocó en 23 pacientes que presentaron hemorragia posparto por hipotonía o atonía uterina, y se tuvo una efectividad del 94.4 por ciento luego de un parto vaginal y del $80 \%$ luego de una cesárea. Reafirmando que es una excelente opción en el manejo de este tipo de complicaciones. Conclusiones: La hemorragia posparto es la segunda causa de muerte materna a nivel nacional, el accionar técnico y oportuno ayudará a disminuir la morbimortalidad materna que puede presentar esta patología; la utilización del balón de compresión hidrostático intrauterino es una excelente alternativa previo al manejo quirúrgico de esta patología.

Palabras Clave: hemorragia posparto, hemorragia uterina, taponamiento uterino con balón.

\section{Introducción}

La hemorragia posparto (HPP) es la segunda causa de muerte materna a nivel nacional [1], y una de las principales causas de muerte materna a nivel mundial [2-4], y se la define como la presencia de sangrado de una cantidad mayor a $500 \mathrm{~mL}$ posteriormente a un parto normal o una cantidad mayor a $1000 \mathrm{~mL}$ posteriormente a una cesárea [1, 5-7], o cualquier sangrado independientemente de la cantidad que pueda causar inestabilidad hemodinámica en la paciente $[1,8]$.

Se clasifica en primaria o temprana si se produce dentro de las primeras $24 \mathrm{hr}$ luego del parto; y en secundaria o tardía si se produce luego de las $24 \mathrm{hr}$ hasta las 12 semanas del posparto [9]. Además, cualquier sangrado que supera los valores esperados y llega a producir tanto signos como síntomas característicos de hipovolemia [9-12]. Con relación a la hemorragia posparto primaria la principal causa es la alteración del tono uterino como la hipotonía o atonía uterina que es la falta de contracción del miometrio produciendo flacidez del útero con la consiguiente pérdida sanguínea que puede ser tan importante que puede comprometer la vida de la paciente. Se considera una emergencia obstétrica [13-15] y lo más importante dentro del manejo es reconocer a tiempo la hemorragia activa excesiva para evitar que sea potencialmente mortal, es primordial identificar la causa del sangrado e iniciar a tiempo la intervención adecuada [13].

Dentro del manejo de la hipotonía o atonía uterina como principales causas de HPP está el uso de medicamentos retractores uterinos y cuando no es posible corregir este problema con esos medicamentos se puede realizar el clampeo de las arterias uterinas por vía vaginal y si persiste el sangrado se puede realizar un taponamiento uterino con un balón de compresión hidrostático que actuará ocluyendo las arterias espiraladas abiertas y disminuirá el sangrado existente previo a la decisión del manejo quirúrgico 
de esta patología [8]. Se realizó una investigación sobre la efectividad de la colocación del balón de compresión intrauterino hidrostático en casos de atonía o hipotonía uterina desde octubre de 2015 a septiembre 2016 en el Hospital Provincial General Docente de Riobamba.

\section{Metodología}

En la presente investigación se realizó un estudio no experimental, descriptivo, transversal, prospectivo de la efectividad de la colocación del balón de compresión intrauterino hidrostático como método para corregir la hemorragia posparto en casos de atonía o hipotonía uterina posparto o pos cesárea de octubre 2015 a septiembre 2016 en el Hospital Provincial General Docente de Riobamba.

\section{Resultados}

En número de nacimientos que se produjeron en el Hospital Provincial General Docente de Riobamba entre octubre del 2015 a septiembre del 2016 fueron 3654, de los cuales 2485 nacimientos que representan el 68\% fueron por parto normal y 1169 nacimientos que representan el 32\% fueron por cesárea (Gráfico 1).

De los 3654 nacimientos, 63 casos que representan el 1,7\% se complicaron con hemorragia posparto, de los cuales 18 nacimientos que representan el $78 \%$ de las hemorragias posparto fueron por parto normal y 5 nacimientos que representan el 22\% de estas hemorragias fueron por cesárea (Gráfico 2).

Dentro de las causas de hemorragia posparto tenemos por alteración del tono uterino 43 casos que representa el $68,3 \%$, por trauma 14 casos que representa el $22,2 \%$, por retención de tejido placentario o membranas corioamnióticas 6 casos que representan el 9,5\% y por trastornos de la coagulación 1 caso que representa el 1,58\% (Gráfico 3).

De los 63 nacimientos que se complicaron con hemorragia posparto a 23 pacientes que representan el $36,5 \%$ se les colocó un balón de compresión intrauterina hidrostático, y de estos 18 pacientes que representan el $78 \%$ eran por parto vaginal y 5 pacientes que representan el 22\% eran posterior a una cesárea (Gráfico 4).

De acuerdo a la edad cronológica de las pacientes, la mayoría a quién se le colocó el balón de compresión intrauterino hidrostático estaban entre los 20 a 24 años con 6 casos, que representan el $26 \%$ de todos los casos, le sigue en incidencia pacientes entre 25 a 29 años con 5 casos que representan el $22 \%$ y la menor incidencia se presentó en menores de 15 años con 1 caso que representa el 4\% (Gráfico 5).

De acuerdo a la edad gestacional al nacimiento, 20 casos que representan el $80 \%$ de las HPP y a quienes se colocó el balón de compresión intrauterino hidrostático tenía un embarazo a término entre 37 a 41 6/7 semanas de gestación.

El promedio de sangrado medido con fundas retrosacales que son dispositivos que recolectan la sangre luego de un parto normal o por estimación subjetiva del personal médico es de $1157 \mathrm{~mL}$, con un máximo de $2700 \mathrm{~mL}$ y un mínimo de $600 \mathrm{~mL}$. 
La efectividad del uso del balón de compresión intrauterino hidrostático que se colocó luego de un parto normal fueron 17 casos que representa el 94,4\%, y luego de una cesárea fueron 4 casos que representan el 80\% de efectividad (Gráfico 6).

NACIMIENTOS OCTUBRE 2015 - SEPTIEMBRE

2016

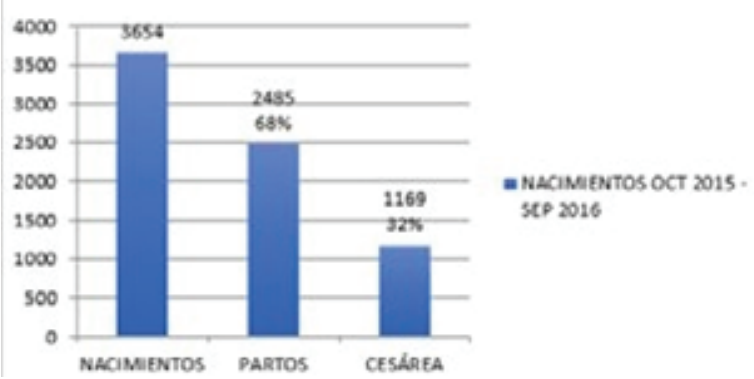

Figure 1

Total de nacimientos en el HPGDR de octubre 2015 a septiembre 2016 y número y porcentaje de partos normales y por cesárea.

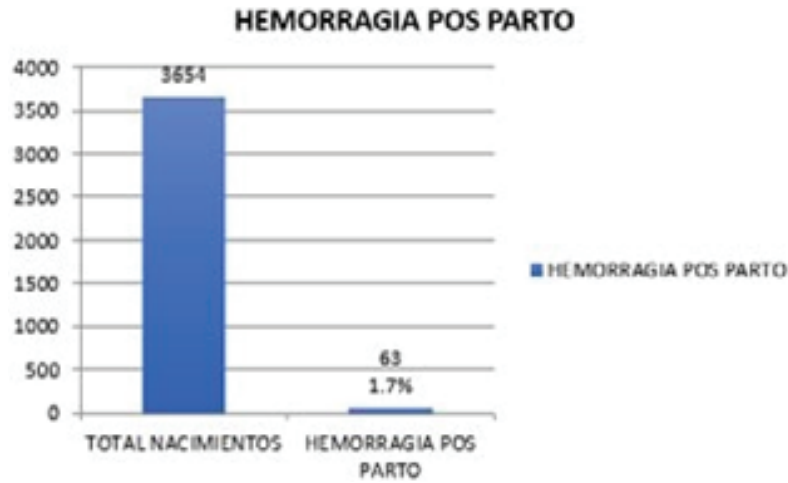

Figure 2

Número y porcentaje de partos que se complicaron con HPP, en el HPGDR de octubre 2015 a septiembre 2016.

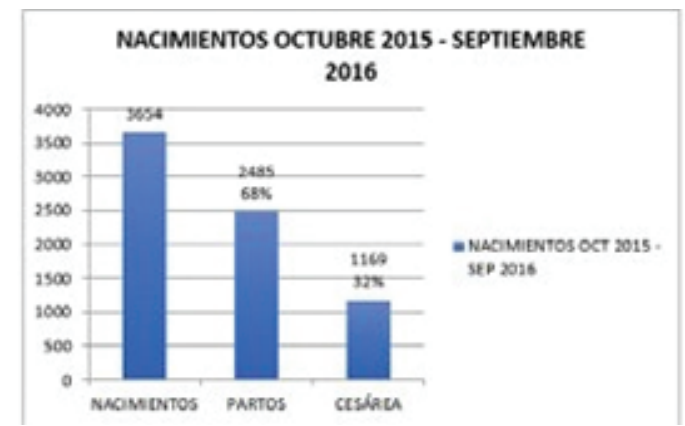

Figure 3

Causas de HPP, en el HPGDR de octubre 2015 a septiembre 2016. 
USO DE BALÓN DE COMPRESIÓN INTRAUTERINA EN CASOS DE HPP

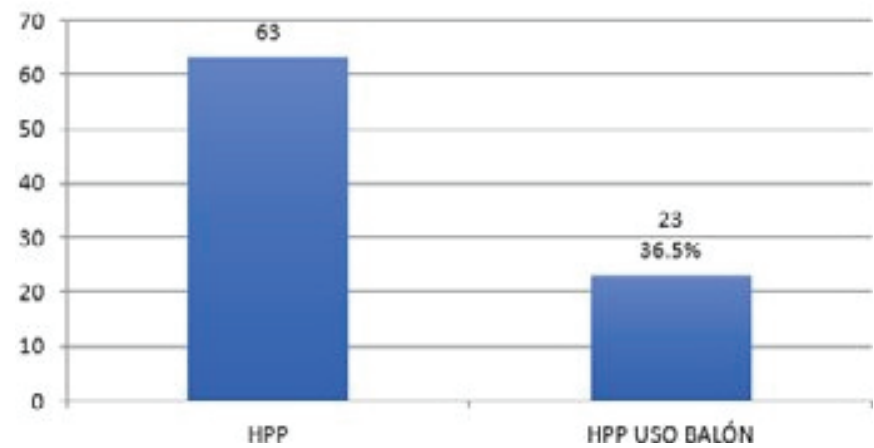

Figure 4

Colocación del balón de compresión intrauterino hidrostático, en el HPGDR de octubre 2015 a septiembre 2016.

COLOCACIÓN DE BALÓN INTRAUTERINO HPGDR

OCTUBRE 2015 - SEPTIEMBRE 2016. POR EDAD

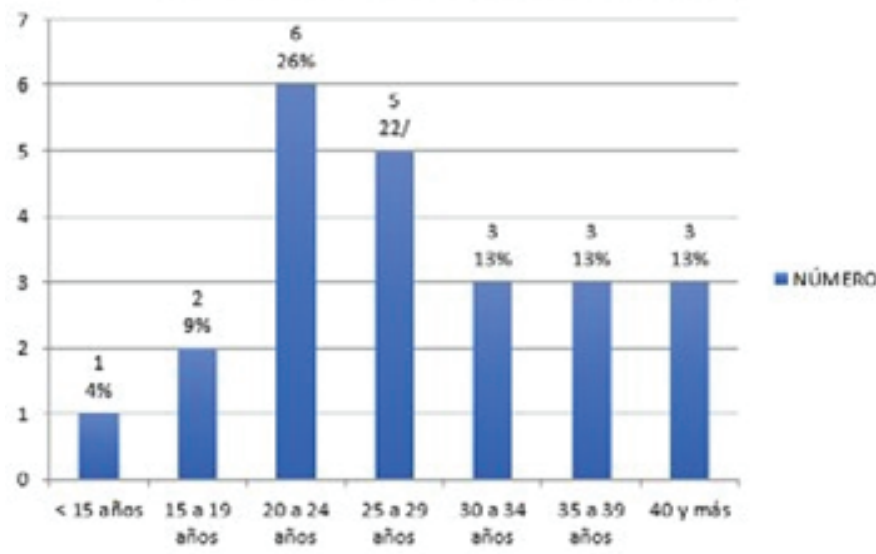

Figure 5

Colocación del balón de compresión intrauterino hidrostático de acuerdo a la edad cronológica de pacientes, en el HPGDR de octubre 2015 a septiembre 2016.

\section{Discusión}

La hemorragia posparto (HPP) es conocida como una emergencia obstétrica que puede producirse posterior a la atención de un parto vaginal o a un parto por cesárea, y dependiendo de su causa se puede realizar múltiples intervenciones médicas y quirúrgicas que pueden ser muy eficaces para su manejo. Lo más importante es saber reconocer de manera temprana el sangrado excesivo para evitar que sea potencialmente mortal, además se debe identificar la causa de base del sangrado e iniciar el manejo oportuno $[16,17]$.

La HPP primaria, es conocida como inmediata o precoz, la cual se presenta en las primeras $24 \mathrm{hr}$ luego del parto y su principal causa es la atonía uterina, que es la incapacidad del útero para contraerse luego de un parto $[1,6,7]$. 


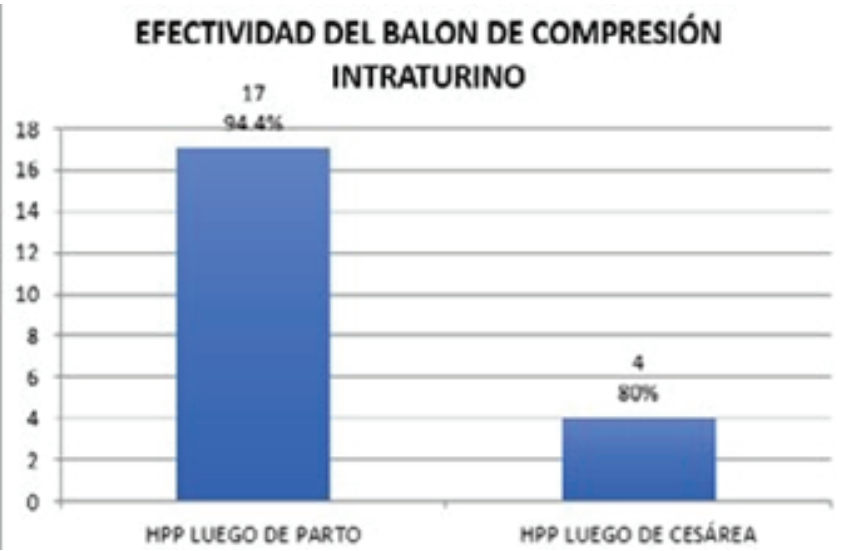

Figure 6

Efectividad del balón de compresión intrauterino hidrostático, en el HPGDR de octubre 2015 a septiembre 2016.

La incidencia de HPP aproximadamente varía del 1 al 5 por ciento de todos los partos [10]. En el estudio realizado en esta investigación representa el 1,7\% que está dentro del rango mencionado a nivel mundial.

El riesgo de presentar una HPP después de un parto es elevado, lo cual se puede producir por un aumento del flujo sanguíneo de la arteria uterina que al final del embarazo puede llegar a ser aproximadamente de un 15\% del gasto cardiaco total que representa de unos 500 a $700 \mathrm{~mL} / \mathrm{min}$ [10]. Por lo tanto, una paciente que presente esta complicación si no es manejada adecuadamente, en poco tiempo puede presentar un choque hipovolémico y aumentar la morbimortalidad.

La alteración de los signos vitales como la disminución de la tensión arterial comúnmente no se presenta hasta que se produce una pérdida de alrededor del $25 \%$ de la volemia corporal total (mayor o igual a $1500 \mathrm{~mL}$ en el embarazo), posterior a esta cantidad puede aparecer signos bajos la tensión arterial y un aumento la frecuencia cardíaca por minuto [10].

El manejo adecuado de la HPP es dependiente de la cantidad de volemia perdida y su estabilidad hemodinámica [18].

Cuando la causa es la atonía uterina y si luego de la administración de retractores uterinos como la oxitocina, misoprostol, metilergonovina, entre otros no da resultado, una medida eficaz es la colocación del balón de compresión intrauterino hidrostático.

El manejo precoz realizado con un taponamiento uterino con el balón de compresión hidrostático es una intervención que puede llegar a salvar vidas [19-21].

El taponamiento intrauterino realizado con balón se lo puede llegar a realizar en el caso de que los fármacos uterotónicos y la compresión uterina bimanual no logran controlar el sangrado de manera eficaz [22]. El uso del balón de compresión hidrostático se lo realiza antes de recurrir a procedimientos quirúrgicos más invasivos que llegan requerir laparotomía $[17,19]$.

Existe diferentes tipos de dispositivos intrauterinos que se pueden utilizar como balones de compresión hidrostática. 
Balón de compresión intrauterina de Bakri: Es un dispositivo intrauterino compuesto de silicona y es el primer sistema diseñado específicamente para el manejo de la hemorragia posparto [23], la cantidad de líquido recomendada para producir la presión hidrostática interna es de 300 a 500 mL [19, 24]. El globo de silicona colapsado se introduce en el útero y este se llena de líquido, preferiblemente normotérmico y el balón se adapta a la cavidad uterina para taponar el sangrado uterino usando la presión hidrostática generada por el líquido que se administró al balón.

BT-Cath: Es un dispositivo de silicona con una forma de pera invertida que sirve para que se adapte a la forma de la cavidad uterina, la cantidad de líquido recomendada para producir la presión hidrostática interna es de $500 \mathrm{~mL}$ [19].

Sistema de taponamiento uterino completo: Es un sistema de doble balón de poliuretano, el un balón se coloca dentro del útero y su llenado es con $750 \mathrm{~mL}$ y otro balón se coloca en vagina y se llena con $300 \mathrm{~mL}$ [19]. Otros dispositivos que se han utilizado para el taponamiento uterino utilizando presión hidrostática son:

Tubo Sengstaken-Blakemore que se utiliza generalmente para el tratamiento de las varices esofágicas sangrantes [25].

Sonda vesical de Foley que se utiliza generalmente para drenaje vesical, pero se ha colocado dentro del útero individual o múltiple [26].

Globo urológico de Rusch que se utiliza para estiramiento de la vejiga [25].

Sonda vesical de Foley a la cual en su extremo medio y distal se anuda un condón con hilo de sutura para evitar que escape el líquido que se administrará, la recomendación es que se llene el preservativo con $500 \mathrm{~mL}$. De líquido, el condón adopta la forma de la cavidad uterina y por presión hidrostática generada por el líquido se ocluye los vasos sangrantes y se corrige la hemorragia posparto [20, 27, 28].

Colocación de un guante quirúrgico en lo posible No 8 que se anuda a una sonda de Foley y se introduce dentro de la cavidad uterina, se llena con líquido máximo hasta $500 \mathrm{~mL}$ [25].

El Hospital Provincial General Docente de Riobamba (HPGDR), es un hospital de segundo nivel de atención que pertenece al Ministerio de Salud Pública (MSP), está ubicado en la ciudad de Riobamba, provincia de Chimborazo, es un hospital de referencia tanto provincial como zonal, principalmente de la zona 3 del país, al que pertenecen las provincias de Chimborazo, Tungurahua, Cotopaxi y Pastaza.

El trabajo de investigación que se realizó, cuyo objetivo fue determinar la efectividad de la utilización del balón intrauterino en casos de hemorragia posparto por atonía o hipotonía uterina en el Hospital Provincial General Docente de Riobamba de Octubre del 2015 a Septiembre del 2016, se utilizó como dispositivo de taponamiento intrauterino un preservativo que se fijó a una sonda de Foley No 16 con un hilo de sutura, generalmente poliglactina del número 0 [22] y se administró $500 \mathrm{~mL}$ de solución salina normal a este dispositivo.

El mecanismo de acción de estos dispositivos intrauterinos es provocar una presión hidrostática dentro del útero generada por la administración del líquido infundido al dispositivo [19] y así provocar oclusión de los vasos sanguíneos espiralados que por 
falta de contracción del músculo uterino por la atonía o hipotonía uterina provocan el sangrado.

Esta presión hidrostática puede o no ser superior a la tensión arterial sistémica [29, 30]. El objetivo es reducir el flujo sanguíneo y facilitar la hemostasia por la compresión de los vasos sanguíneos [19].

Pero, si el sangrado persiste a pesar de la colocación del dispositivo de taponamiento intrauterino, se deben realizar procedimientos oportunos para un tratamiento definitivo, que puede incluir la necesidad de embolización arterial o una intervención quirúrgica por laparotomía [19].

El balón de compresión intrauterino hidrostático que se utilizó en esta investigación se elaboró con un preservativo anudado con hilo de sutura una sonda de Foley No 16 y se administró $500 \mathrm{~mL}$ de solución salina normal a través de un equipo de venoclisis y utilizando un infusor o tensiómetro para la aumentar la perfusión hacia el preservativo y lograr la compresión hidrostática para corregir la HPP.

\subsection{Técnica}

- Preparación del balón de compresión intrauterino hidrostático.

- Colocación de una sonda de Foley vesical para vaciar vejiga y como soporte para determinar la diuresis de la paciente.

- Antibiótico profilaxis, generalmente se utilizó cefazolina $2 \mathrm{~g}$ intravenoso antes del procedimiento y posteriormente $1 \mathrm{~g}$ intravenoso cada $8 \mathrm{hr}$ por $24 \mathrm{hr}$ [19].

- Asepsia y antisepsia perineal, vulvar, vaginal y cervical con yodopovidona.

- Colocación de valva vaginal posterior y pinzamiento del cérvix, labio anterior.

- Colocación del balón de compresión intrauterino hidrostático.

- Mantener el goteo de oxitocina durante el tiempo que se mantenga colocado el balón de compresión intrauterino.

- El balón se mantiene hasta por 24 hr y cuando se va a retirar se descomprime $100 \mathrm{~mL}$ cada $30 \mathrm{~min}$.

No existen ensayos aleatorios que evalúen el uso de estos dispositivos ni comparen su efectividad de manera adecuada, pero las tasas de éxito del balón intrauterino reportada en diferentes estudios para controlar la HPP, oscilan entre 90 a 100 por ciento después del parto vaginal y del 20 y 65 por ciento después del parto por

cesárea [19]. La cual es similar a la encontrada en la investigación realizada en el HPGDR que fue del 94,4\% luego de un parto normal y del $80 \%$ luego de una cesárea.

\section{Conclusiones}

La preparación del balón de compresión intrauterino utilizando un preservativo y una sonda de Foley es fácil de realizar. 
Es una de las principales alternativas para el manejo de la HPP, principalmente por atonía o hipotonía uterina, luego de la utilización de medicamentos retractores uterinos y si estos no han sido efectivos, y previo la probable solución quirúrgica por laparotomía de este cuadro clínico.

La efectividad encontrada en este estudio es muy similar a la encontrada en otros estudios realizados.

Se debe dentro de la formación médica profesional capacitar a los estudiantes y profesionales de la salud sobre la preparación de este dispositivo y se debe dar entrenamiento en la utilización del mismo en caso de HPP.

\section{Agradecimientos}

Agradezco a todos los autores de este trabajo que han colaborado eficientemente en esta investigación que tiene como base determinar la efectividad del uso del balón de compresión intrauterino en casos de HPP en el periodo de octubre de 2015 a septiembre 2016 en el HPGDR, también a la Facultad de Salud Pública y a la Carrera de Medicina de la Escuela Superior Politécnica de Chimborazo por realizar este congreso y publicar esta investigación que es una fuente de información adecuada que nos permite actualizarnos y compartir conocimientos e investigaciones para realizar de una mejor manera nuestro quehacer médico.

\section{Conflicto de Intereses}

Los autores declaran que no existen.

\section{Limitación de Responsabilidad}

Los puntos de vista realizados y mostrados en este trabajo de investigación son de responsabilidad de los autores quienes han realizado la investigación, sin ningún cargo a responsabilidad de la institución en la que laboran los autores.

\section{Fuentes de Apoyo}

Los autores declaramos que las fuentes de apoyo y financiación que permitieron realizar esta investigación son

propios.

\section{References}

[1] León W, Villamarín S, Velasco S, González F, Aguinaga G. Prevención, diagnóstico y tratamiento de la hemorragia posparto. Guía de Práctica Clínica. Quito: Ministerio de Salud Pública; 2013. Available from: http://www.salud.gob.ec/wp-content/uploads/2014/05/Guía-de-hemorragia-postparto.pdf 
[2] Khan KS, Wojdyla D, Say L, Gülmezoglu AM, Van Look PF. WHO analysis of causes of maternal death: A systematic review. Lancet. 2006;367(9516):1066-74.

[3] Francois KE, Foley MR. Obstetricia Embarazos normales y de riesgo. Séptima. Elsevier; 2019 Hemorragia preparto y posparto. Available from: https://www.clinicalkey.es/service/content/pdf/ watermarked/3-s2.0-B978849113358200018X.pdf?locale=es_ES\&searchIndex=

[4] Belfort M. Overview of postpartum hemorrhage. UpToDate. 2020. Available from: https://www.uptodate. com/contents/overview-of-postpartum-hemorrhage/print?search=hemorragiaposparto\&source= search_result\&selectedTitle=1\$\{lsim $\} \$ 150 \&$ usage_type=default\&display_rank $=1$

[5] Belfort M. Overview of postpartum hemorrhage. UpToDate. 2015;1. Available from: https: //www.uptodate.com/contents/overview-of-postpartum-hemorrhage?search=atoniauterina\&source= search_result\&selectedTitle=1\$\{lsim\}\$51\&usage_type=default\&display_rank=1

[6] Secretaría Distrital de Salud. Guía de hemorragia posparto. Código Rojo. 2014. Available from: http: //www.saludcapital.gov.co/DDS/Publicaciones/GuiaMaternidad-CodigoRojo_7A.pdf

[7] Resendiz F, Castillo D, Orozco F, et al. Diagnóstico y tratamiento del choque hemorrágico en Obstetricia. Guía de Evidencias y Recomendaciones: Guía de Práctica Clínica. México: CENETEC; 2017. Available from: www.cenetec.salud.gob.mx

[8] López N, Pérez Á, De la Torre T, Nieves R, et al. Prevención y Manejo de la Hemorragia Postparto en el primero, segundo y tercer nivel de atención. Resumen de Evidencias y Recomendaciones: Guía de Práctica Clínica [Internet]. Secretaría de Salud CENETEC. México; 2018. Available from: http://www. cenetec-difusion.com/CMGPC/SS-103-08/ER.pdf

[9] Committee on Practice Bulletins-Obstetrics. Postpartum Hemorrhage. Clinical Management Guidelines for Obstetrician- Gynecologists. Am Coll Obstet Gynecol. 2017;130(76).

[10] Belfort M. Overview of postpartum hemorrhage. UpToDate. 2018. Available from: http://content. wkhealth.com/linkback/openurl?sid=WKPTLP:landingpage\&an=00006250-201405000-00013

[11] Menard K, Main E, Currigan S. Executive Summary of the reVITALize Initiative. Obstet Gynecol. 2014;129(4):603-7.

[12] Berens P. Overview of the postpartum period: Physiology, complications, and maternal care. UpToDate. 2018. Available from: https:/www.uptodate.com/contents/ overview-of-the-postpartum-period-physiology-complications-and-maternal-care/print? search=hemorragiaposparto\&source=search_result\&selectedTitle=5\$\{sim $\} \$ 150 \&$ usage_type= default\&display_rank=5

[13] Belfort M. Pospartum hemorrhage: Medical and minimally invasive management. UpToDate. 2018. Available from: https://www.uptodate.com/contents/ postpartum-hemorrhage-medical-and-minimally-invasive-management/print?search= hemorragiaposparto\&source=search_result\&selectedTitle=3\$\{lsim\}\$150\&usage_type= default\&display_rank=3

[14] Belfort M. Postpartum hemorrhage: Management approaches requiring laparotomy. UpToDate. 2018. Available from: https://www.uptodate.com/contents/ postpartum-hemorrhage-management-approaches-requiring-laparotomy/print?search= hemorragiaposparto\&source=search_result\&selectedTitle=4\$\{lsim $\} \$ 150 \&$ usage_type= default\&display_rank=4

[15] Belfort M. Summary of postpartum hemorrhage. UpToDate. 2017;1-29.

[16] Belfort M. Postpartum hemorrhage: Medical and minimally invasive management. UpToDate. 2019 Available from: https://www.uptodate.com/contents/ postpartum-hemorrhage-medical-and-minimally-invasive-management/print?sectionName= Transfuseredbloodcells, platelets, plasma\&search=hemorragiapostparto\&topicRef=6710\&anchor= H7\&source=see_link

[17] Belfort M. Management of postpartum hemorrhage in vaginal delivery. UpToDate. 2015.

[18] Belfort M. Management of postpartum hemorrhage in vaginal delivery. UpToDate. 2017;

[19] Tolosa J, Bakri Y, Arulkumaran S. Intrauterine balloon tamponade for control of postpartum hemorrhage. UpToDate. 2018. Available from: https://www.uptodate.com/ contents/intrauterine-balloon-tamponade-for-control-of-postpartum-hemorrhage/print? search=hemorragiaposparto\&source=search_result\&selectedTitle=6\$\{sim $\} \$ 150 \&$ usage_type $=$ default\&display_rank=6

[20] Tindell K, Garfinkel R, Abu-Haydar E, et al. Uterine balloon tamponade for the treatment of postpartum haemorrhage in resource-poor settings: A systematic review. BJOG An Int J Obstet Gynaecol. 2013;120(1):5-14. Available from: http://doi.wiley.com/10.1111/j.14710528.2012.03454.x

[21] Belfort M. Postpartum hemorrhage: Medical and minimally invasive management. UpToDate. 2020. Available from: https://www.uptodate.com/contents/ postpartum-hemorrhage-medical-and-minimally-invasive-management/print?search= 
hemorragiaposparto\&source=search_result\&selectedTitle=3 150\&usage_type=default\&display_ rank=3

[22] Calle J, Ríos P, Yuen Chon V, Rojas L, Rivadeneira J, Pareja C. Score MAMÁ y claves obstétricas. Protocolo. $1^{\text {st }}$ ed. Quito: Ministerio de Salud Pública del Ecuador; 2017.

[23] Bakri Y. Balloon device for control of obstetrical bleeding. Eur J Obs Gynecol Reprod Biol. 1999;86(S84).

[24] Kaya B, Tuten A, Daglar K, et al. Balloon tamponade for the management of postpartum uterine hemorrhage. J Perinat Med. 2014;42(6):745-53.

[25] Bakri Y, Arulkumaran S. Intrauterine balloon tamponade for the control of postpartum haemorrhage. UpToDate. 2015.

[26] Rathore AM, Gupta S, Manaktala U, Gupta S, Dubey C, Khan M. Uterine tamponade using condom catheter balloon in the management of non-traumatic postpartum hemorrhage. J Obstet Gynaecol Res. 2012;38(9):1162-7.

[27] Thapa K, Malla B, Pandey S, Amatya S. Intrauterine condom tamponade in management of post partum haemorrhage. J Nepal Health Res Counc. 2010;8(1):19-22.

[28] Akhter S, Begum MR, Kabir Z, Rashid M, Laila TR, Zabeen F. Use of a condom to control massive postpartum hemorrhage. MedGenMed. 2003;5(3):38.

[29] Anger HA, Dabash R, Durocher J, et al. The effectiveness and safety of introducing condom-catheter uterine balloon tamponade for postpartum haemorrhage at secondary level hospitals in Uganda, Egypt and Senegal: A stepped wedge, cluster-randomised trial. BJOG. 2019;126(13):1612-21.

[30] Kong C, To W. Intraluminal pressure of uterine balloon tamponade in the management of severe postpartum hemorrhage. J Obs Gynaecol Res. 2018; 